NISTIR 7890

\title{
NIST/NIH Vitamin D Metabolites Quality Assurance Program Report of Participant Results: Winter 2010 Comparability Study (Exercise 1)
}

Mary Bedner

Katrice A. Lippa

http://dx.doi.org/10.6028/NIST.IR.7890

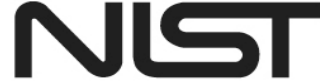

National Institute of Standards and Technology U.S. Department of Commerce 
NISTIR 7890

\title{
NIST/NIH Vitamin D Metabolites Quality Assurance Program Report of Participant Results: Winter 2010 Comparability Study (Exercise 1)
}

\author{
Mary Bedner \\ Katrice A. Lippa \\ Chemical Sciences Division \\ Material Measurement Laboratory
}

http://dx.doi.org/10.6028/NIST.IR.7890

December 2012

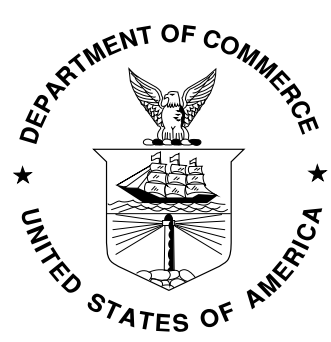

U.S. Department of Commerce Rebecca Blank, Acting Secretary 


\section{ABSTRACT}

The National Institute of Standards and Technology (NIST) has established a Vitamin D Metabolites Quality Assurance Program in collaboration with the National Institutes of Health (NIH) Office of Dietary Supplements (ODS). For this first pilot exercise (Winter 2010 Comparability Study), participants were asked to use the methodology of their choice to measure concentrations of 25-hydroxyvitamin D in control and study materials distributed by NIST. The study material consisted of triplicate samples of SRM 1950 Metabolites in Human Plasma. SRM 2972, which is comprised of separate ethanolic calibration solutions with known concentrations of $25(\mathrm{OH}) \mathrm{D}_{2}$ and $25(\mathrm{OH}) \mathrm{D}_{3}$, was provided as a control material. Participants provided their data to NIST, where it was compiled and evaluated for trueness relative to the NIST value, within-laboratory precision, and concordance within the participant community. A report of results was provided to all participants of the exercise, and laboratories were identified by code numbers known only to them. The results from this first pilot exercise are reported along with a summary of the analytical methods used. 


\section{OVERVIEW OF WINTER 2010 COMPARABILITY STUDY}

For the Winter 2010 Comparability Study (Exercise 1) of the NIST/NIH Vitamin D Metabolites Quality Assurance Program (VitDQAP), control and human serum study samples were distributed to participants for evaluation. SRM 2972, which is comprised of separate ethanolic solutions with known concentrations of 25-hydroxyvitamin $\mathrm{D}_{2}\left(25(\mathrm{OH}) \mathrm{D}_{2}\right)$ and 25hydroxyvitamin $\mathrm{D}_{3}\left(25(\mathrm{OH}) \mathrm{D}_{3}\right)$, was provided as a control material for assay calibration or verification. Participants were asked to provide single results for each of these solutions. In addition, participants were asked to determine $25(\mathrm{OH}) \mathrm{D}_{2}, 25(\mathrm{OH}) \mathrm{D}_{3}$, and a total concentration of 25-hydroxyvitamin $\mathrm{D}\left(25(\mathrm{OH}) \mathrm{D}_{\text {Total }}=25(\mathrm{OH}) \mathrm{D}_{2}+25(\mathrm{OH}) \mathrm{D}_{3}\right)$ in each of three vials $\mathrm{A}, \mathrm{B}$, and C, which were triplicate samples of SRM 1950 Metabolites in Human Plasma.

In the Winter 2010 exercise, there were a total of 16 participants and 17 datasets (one participant provided data for two different methods). Ten of these datasets originated from liquid chromatographic (LC) methods; of those, 9 were from LC with tandem mass spectrometric detection (LC-MS/MS) and 1 was from LC with ultraviolet absorbance detection (LC-UV). Seven datasets originated from immunoassay (IA) techniques, including 3 from enzyme immunoassay (EIA), 1 from chemiluminescence immunoassay (CLIA), and 3 from radioimmunoassay (RIA). Appendices A-1 and A-2 summarize the immunoassay and LC methods, respectively, used by the participants.

The raw data received from all participants is summarized in Appendix B. The data include the results for $25(\mathrm{OH}) \mathrm{D}_{2}, 25(\mathrm{OH}) \mathrm{D}_{3}$ and $25(\mathrm{OH}) \mathrm{D}_{\text {Total }}$ in the three vials $(\mathrm{A}, \mathrm{B}$ and C) of SRM 1950 (study sample) and for $25(\mathrm{OH}) \mathrm{D}_{2}$ and $25(\mathrm{OH}) \mathrm{D}_{3}$ in the ethanolic calibration solutions. Only the LC-based methods could distinguish between 25(OH) $\mathrm{D}_{2}$ and 25(OH) $\mathrm{D}_{3}$ in the SRM 1950 samples. All 10 of the LC-based datasets reported values for $25(\mathrm{OH}) \mathrm{D}_{3}$, but only two reported values for $25(\mathrm{OH}) \mathrm{D}_{2}$. The amount of $25(\mathrm{OH}) \mathrm{D}_{2}$ in $\mathrm{SRM} 1950$ was very low and below the detection limit for most of the LC-based methods. The seven datasets from the immunoassay methods could not distinguish between $25(\mathrm{OH}) \mathrm{D}_{2}$ and $25(\mathrm{OH}) \mathrm{D}_{3}$ and reported the $25(\mathrm{OH}) \mathrm{D}_{\text {Total }}$ in SRM 1950 (study sample). However, both LC and immunoassay datasets provided individual values for $25(\mathrm{OH}) \mathrm{D}_{2}$ and $25(\mathrm{OH}) \mathrm{D}_{3}$ in the ethanolic controls because the analytes were in separate solutions.

Appendix B also provides the summarized NIST results for both SRM 1950 (study material) and SRM 2972 (controls). 


\section{WINTER 2010 EXERCISE RESULTS AND DISCUSSION}

\section{5(OH) $D_{2}$ and 25(OH) $D_{3}$ in the control solutions (SRM 2972)}

A summary of the individual participant data for $25(\mathrm{OH}) \mathrm{D}_{2}$ and $25(\mathrm{OH}) \mathrm{D}_{3}$ in the SRM 2972 control solutions is provided in Table 1. The community results are summarized at the bottom of the table for all reported methods and the LC methods only. The community results include the total number of quantitative values reported $(\mathrm{N})$, the median value, the MADe (the median absolute deviation estimate, a robust estimate of the standard deviation), and the percent coefficient of variation (CV\%). Consensus statistics were not calculated for the data from the IA methods because of the limited number of data reported $(\mathrm{N}=3)$. Table 1 also presents the NIST certified values with expanded uncertainties corresponding to 95\% confidence.

For the ethanolic control solutions (SRM 2972), the single data values for $25(\mathrm{OH}) \mathrm{D}_{2}$ and $25(\mathrm{OH}) \mathrm{D}_{3}$ reported by each individual laboratory are plotted in Figures 1a and $\mathbf{1 b}$, respectively. The two primary methods of analysis (LC and immunoassay) are displayed separately with closed $(\bullet)$ and open $(\circ)$ circles, respectively. For each of these graphs, the black solid line

(-) represents the consensus median, and the black dotted lines (- - - -) represent the consensus variability $(2 \times \mathrm{MADe})$. The laboratories with results that fall between the two dotted lines are within the consensus variability area. The NIST-assessed values of $238.6 \mathrm{ng} / \mathrm{mL} \pm 3.9$ $\mathrm{ng} / \mathrm{mL}$ and $334.8 \mathrm{ng} / \mathrm{mL} \pm 5.2 \mathrm{ng} / \mathrm{mL}$ for $25(\mathrm{OH}) \mathrm{D}_{2}$ and $25(\mathrm{OH}) \mathrm{D}_{3}$, respectively, for this control material (SRM 2972) are provided by red squares ( $\square$ ) (with error bars representing $\pm U_{95}$ ). 
Table 1. Summary of participant data for $25(\mathrm{OH}) \mathrm{D}_{2}(\mathrm{ng} / \mathrm{mL})$ and $25(\mathrm{OH}) \mathrm{D}_{3}(\mathrm{ng} / \mathrm{mL})$ in the SRM 2972 control solutions.

\begin{tabular}{|c|c|c|}
\hline \multicolumn{3}{|c|}{$25(\mathrm{OH}) \mathrm{D}_{2}(\mathrm{ng} / \mathrm{mL})$} \\
\hline & & SRM 2972 \\
\hline $\mathrm{Lab}$ & Method & Value \\
\hline 056 & LC-MS/MS & 211.3 \\
\hline 060 & LC-MS/MS & 235.0 \\
\hline 062 & RIA & 175.7 \\
\hline 160 & LC-MS/MS & 237.0 \\
\hline 182 & LC-MS/MS & 262.0 \\
\hline $183 a$ & LC-MS/MS & 161.2 \\
\hline $183 b$ & CLIA & 205.0 \\
\hline 184 & LC-MS/MS & 223.0 \\
\hline $185 a$ & LC-MS/MS & 201.0 \\
\hline 186 & LC-MS/MS & 240.6 \\
\hline 187 & LC-MS/MS & 214.0 \\
\hline 191 & RIA & 309.6 \\
\hline on & $\mathrm{N}$ & 12 \\
\hline$=\overline{0}$ & Median & 218.5 \\
\hline$\varangle \frac{5}{0}$ & MADe & 26.7 \\
\hline & CV\% & 12.2 \\
\hline$\frac{n}{0}$ & $\mathrm{~N}$ & 9 \\
\hline $0 \stackrel{8}{0}$ & Median & 223.0 \\
\hline 」 & MADe & 20.8 \\
\hline 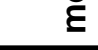 & CV\% & 9.3 \\
\hline & NIST Value & 238.6 \\
\hline & $U_{95}$ & 3.9 \\
\hline
\end{tabular}

\begin{tabular}{|c|c|c|}
\hline \multicolumn{3}{|c|}{$25(\mathrm{OH}) \mathrm{D}_{3}(\mathrm{ng} / \mathrm{mL})$} \\
\hline & & SRM 2972 \\
\hline Lab & Method & Value \\
\hline 056 & LC-MS/MS & 260.4 \\
\hline 060 & LC-MS/MS & 324.2 \\
\hline 062 & RIA & 340.3 \\
\hline 160 & LC-MS/MS & 329.0 \\
\hline 182 & LC-MS/MS & 368.0 \\
\hline $183 a$ & LC-MS/MS & 353.8 \\
\hline $183 b$ & CLIA & 291.0 \\
\hline 184 & LC-MS/MS & 351.0 \\
\hline $185 a$ & LC-MS/MS & 354.4 \\
\hline 186 & LC-MS/MS & 333.8 \\
\hline 187 & LC-MS/MS & 347.0 \\
\hline 191 & RIA & 319.8 \\
\hline & $\mathrm{N}$ & 12 \\
\hline & Median & 337.1 \\
\hline & MADe & 22.8 \\
\hline & CV\% & 6.8 \\
\hline & $\mathrm{N}$ & 9 \\
\hline & Median & 347.0 \\
\hline & MADe & 19.6 \\
\hline & CV\% & 5.6 \\
\hline & NIST Value & 334.0 \\
\hline & & 5.2 \\
\hline
\end{tabular}


Figure 1. Winter 2010 exercise results for (a) $25(\mathrm{OH}) \mathrm{D}_{2}$ and (b) $25(\mathrm{OH}) \mathrm{D}_{3}$ in the ethanolic control (SRM 2972).

(a) $25(\mathrm{OH}) \mathrm{D}_{2}$

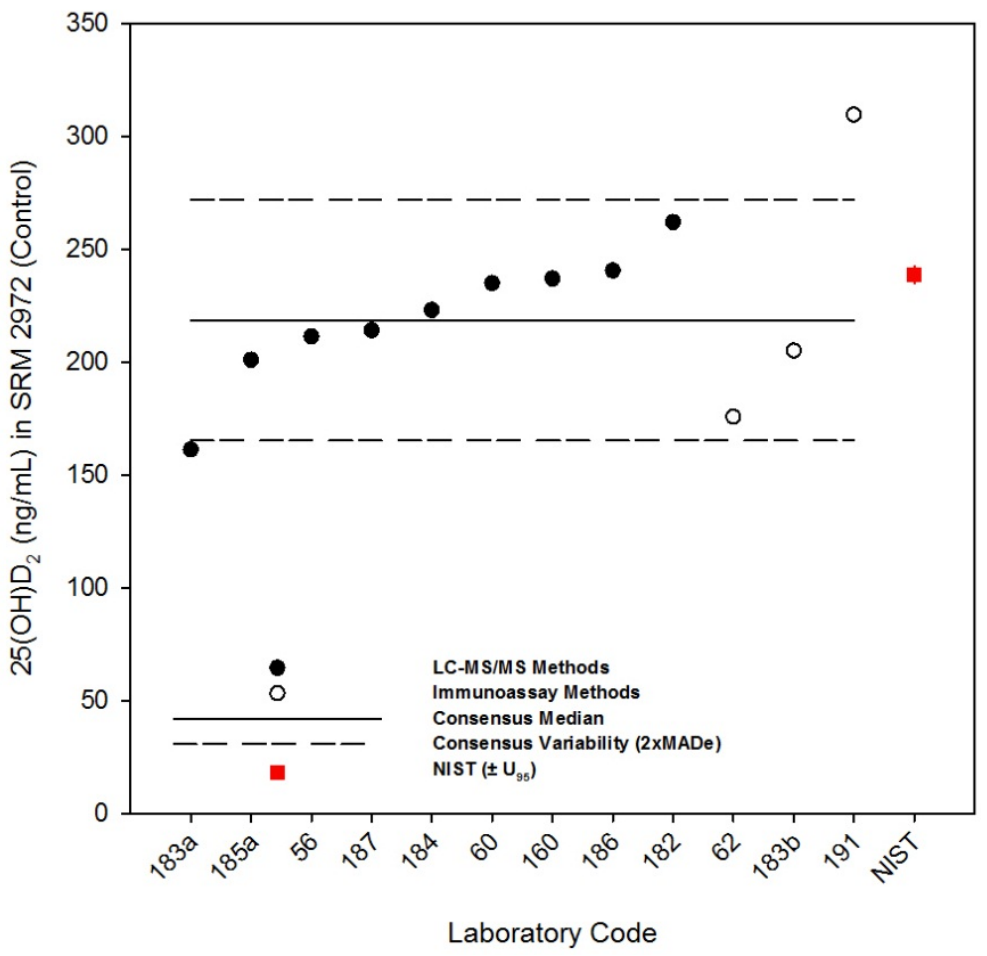

(b) $25(\mathrm{OH}) \mathrm{D}_{3}$

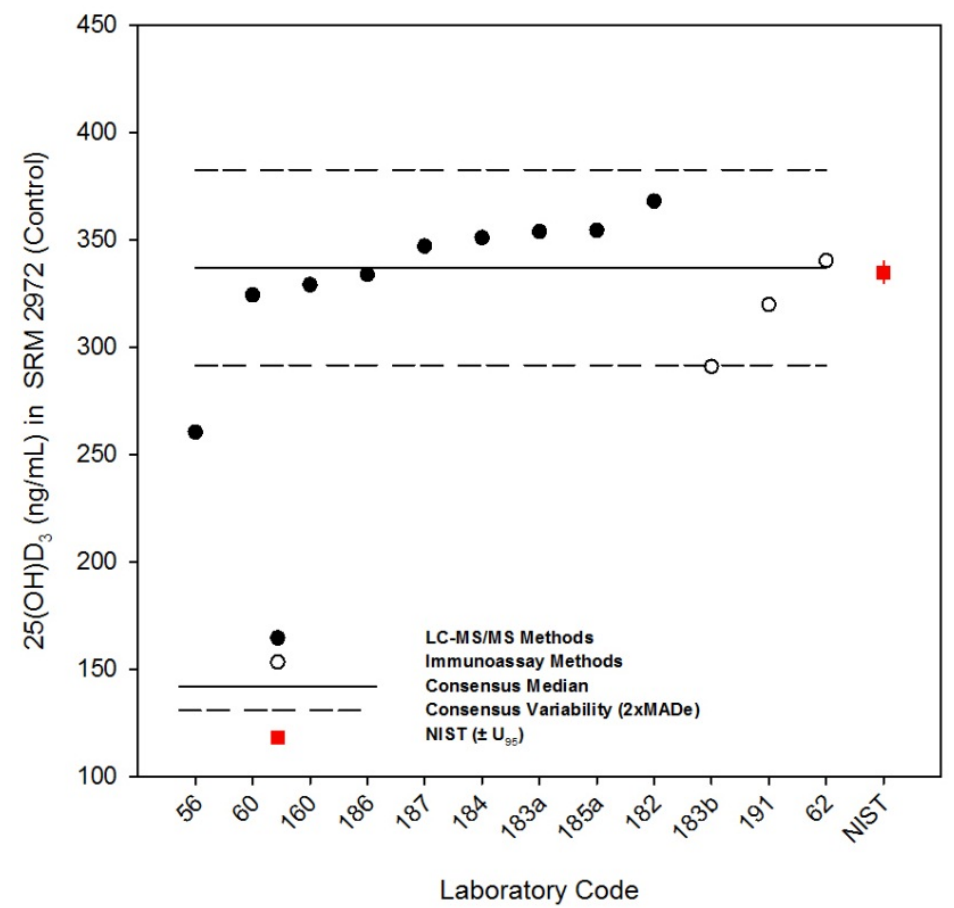




\section{5(OH)D in the study material SRM 1950}

A summary of the individual participant data for $25(\mathrm{OH}) \mathrm{D}_{\text {Total }}$ in SRM 1950 is provided in

Table 2. The summarized data include the mean, standard deviation (SD), and relative percent standard deviation (\%rSD) of the three reported values (vials A, B, and C) for SRM 1950.

The community results are summarized at the bottom of the table for all reported methods, the IA methods only, the LC methods only and the LC-MS/MS methods only. These summarized results include the total number of quantitative values reported, the median value, the MADe, and the percent coefficient of variation.

The table also presents the sum of the NIST certified and reference values for $25(\mathrm{OH}) \mathrm{D}_{3}$ and 25(OH)D $\mathrm{D}_{2}$, respectively, for SRM 1950 (study sample) with approximated 95\% confidence limits $\left(U_{95}\right)$. NIST results for SRM 1950 were obtained using data from both LC-MS and LC-MS/MS techniques; details about the methods and measurements are reported in the Certificate of Analysis for SRM 1950².

For each participant who used an LC method, the calculated average (mean) value and error bars (representing the lab mean value $\pm 2 \times \mathrm{SD}$ ) for $25(\mathrm{OH}) \mathrm{D}_{3}$ in SRM 1950 (study sample) are plotted in Figure 2. The data for $25(\mathrm{OH}) \mathrm{D}_{2}$ are not provided in graphical form because only two laboratories reported values that were above their detection limits. When the error bars for each data point are considered, all laboratory data are within the consensus variability $(2 \times \mathrm{MADe})$.

For all participant datasets, the calculated average (mean) values and error bars (representing the lab mean value $\pm 2 \times \mathrm{SD}$ ) for $25(\mathrm{OH}) \mathrm{D}_{\text {Total }}$ in SRM 1950 (study sample) are plotted in Figure 3 . From the mean values for all datasets, the consensus median and the consensus variability $(2 \times$ MADe) were determined. When the error bars for each data point are considered, all laboratory data are within the consensus variability. Both primary methods of analysis (LC, immunoassay) provide similar mean values and uncertainties for SRM 1950 (study sample).

The precision (as \%rSD) for the three replicate analyses of SRM 1950 (study sample) ranged from $1 \%$ to $10 \%$ for the individual laboratories, where over $2 / 3$ of the laboratories that participated had method precision under 5\%. When all reported data for SRM 1950 (study sample) were considered, the consensus variability was $\approx 14 \%$ and the consensus median was biased $\approx 7 \%$ higher than the NIST-assessed value for this material. A goal for this program is to determine the cause of any bias and to achieve better agreement between the consensus median value and the NIST-assessed value. While the precision of most individual laboratories was acceptable, another program goal is to reduce the consensus variability to better represent the community's measurement capability while also recognizing that a 'fit-for-purpose' variabilitylevel may exist. One approach to meet this goal is to strive for within-laboratory precision of less than $5 \%$ for all participants.

\footnotetext{
${ }^{1}$ https://www-s.nist.gov/srmors/view_cert.cfm?srm=1950
} 
Table 2. Summary of participant data for 25(OH)D $\mathrm{D}_{\text {Total }}(\mathrm{ng} / \mathrm{mL})$ in SRM 1950.

\begin{tabular}{|c|c|c|c|c|c|c|c|}
\hline & \multirow[b]{2}{*}{ Method } & \multirow{2}{*}{$\begin{array}{c}\text { SRM } 1950 \\
\text { Vial A }\end{array}$} & \multirow{2}{*}{$\frac{\text { SRM } 1950}{\text { Vial B }}$} & \multirow{2}{*}{$\frac{\text { SRM } 1950}{\text { Vial C }}$} & \multicolumn{3}{|c|}{ SRM 1950 Combined } \\
\hline $\mathrm{Lab}$ & & & & & Mean & SD & $\%$ rSD \\
\hline 056 & LC-MS/MS & 24.9 & 24.2 & 24.3 & 24.5 & 0.4 & 1.5 \\
\hline 060 & LC-MS/MS & 26.5 & 26.7 & 27.1 & 26.8 & 0.3 & 1.1 \\
\hline 062 & RIA & 22.6 & 24.3 & 23.4 & 23.4 & 0.9 & 3.6 \\
\hline 160 & LC-MS/MS & 26.3 & 29.2 & 27.3 & 27.6 & 1.5 & 5.3 \\
\hline 180 & RIA & 25.4 & 27.0 & 25.5 & 26.0 & 0.9 & 3.5 \\
\hline 182 & LC-MS/MS & 24.0 & 26.0 & 29.0 & 26.3 & 2.5 & 9.6 \\
\hline $183 a$ & LC-MS/MS & 31.3 & 29.8 & 29.3 & 30.1 & 1.0 & 3.5 \\
\hline $183 b$ & CLIA & 30.0 & 29.1 & 29.8 & 29.6 & 0.5 & 1.6 \\
\hline 184 & LC-MS/MS & 23.8 & 28.3 & 27.1 & 26.4 & 2.3 & 8.8 \\
\hline $185 a$ & LC-MS/MS & 26.4 & 26.7 & 27.0 & 26.7 & 0.3 & 1.3 \\
\hline 186 & LC-MS/MS & 27.6 & 25.3 & 23.5 & 25.5 & 2.0 & 8.0 \\
\hline 187 & LC-MS/MS & 25.5 & 24.8 & 26.2 & 25.5 & 0.7 & 2.7 \\
\hline 188 & CLIA & 28.0 & 28.0 & 25.0 & 27.0 & 1.7 & 6.4 \\
\hline 189 & LC-UV & 29.8 & 29.3 & 28.6 & 29.2 & 0.6 & 2.1 \\
\hline 190 & EIA & 32.5 & 30.4 & 30.4 & 31.1 & 1.2 & 3.9 \\
\hline 191 & RIA & 29.9 & 30.4 & 30.1 & 30.1 & 0.2 & 0.8 \\
\hline 192 & EIA & 23.5 & 23.4 & 24.2 & 23.7 & 0.4 & 1.7 \\
\hline & $\mathrm{N}$ & 17 & 17 & 17 & 17 & & \\
\hline$=$ 을 & Median & 26.4 & 27.0 & 27.1 & 26.7 & & \\
\hline 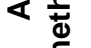 & MADe & 3.5 & 3.2 & 3.1 & 1.8 & & \\
\hline ह & CV\% & 13.2 & 12 & 11 & 7 & & \\
\hline & $\mathrm{N}$ & 7 & 7 & 7 & 7 & & \\
\hline$\varangle \stackrel{8}{\circ}$ & Median & 28.0 & 28.0 & 25.5 & 27.0 & & \\
\hline 二 & MADe & 3.8 & 3.5 & 3.0 & 4.6 & & \\
\hline & CV\% & 13.7 & 13 & 12 & 17 & & \\
\hline & & 10 & 10 & 10 & 10 & & \\
\hline $0 \stackrel{0}{0}$ & Median & 26.3 & 26.7 & 27.1 & 26.6 & & \\
\hline 」吾 & MADe & 2.0 & 2.6 & 1.8 & 1.6 & & \\
\hline & CV\% & 7.6 & 9.7 & 6.6 & 5.9 & & \\
\hline & & 9 & 9 & 9 & 9 & & \\
\hline$\dot{u} \geqq$ & Median & 26.3 & 26.7 & 27.1 & 26.4 & & \\
\hline 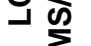 & MADe & 1.9 & 2.4 & 1.3 & 1.3 & & \\
\hline & CV\% & 7.3 & 8.9 & 4.9 & 5.1 & & \\
\hline & NIST Value & 25.30 & 25.30 & 25.30 & 25.30 & & \\
\hline & $U_{95}$ & 0.79 & 0.79 & 0.79 & 0.79 & & \\
\hline
\end{tabular}


Figure 2. Winter 2010 exercise results for $25(\mathrm{OH}) \mathrm{D}_{3}$ using only LC methods in SRM 1950 (study sample).

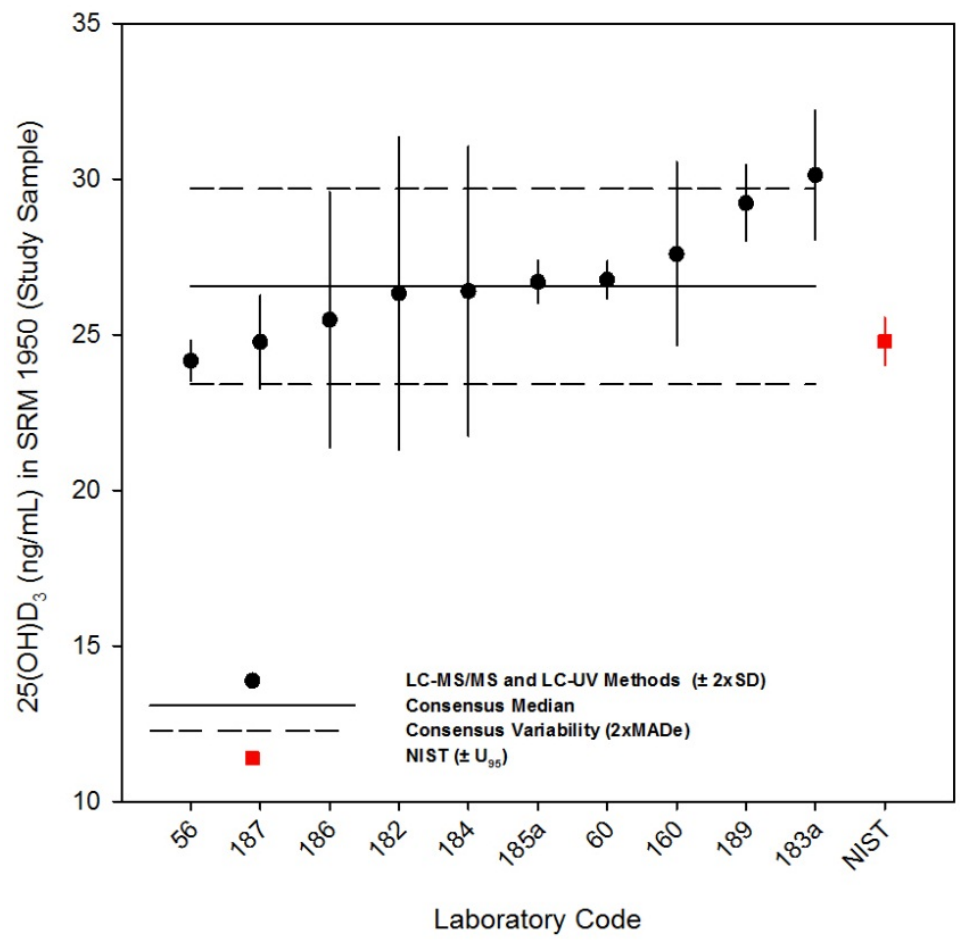

Figure 3. Winter 2010 exercise results for 25(OH)D $\mathrm{D}_{\text {Total }}$ in SRM 1950 (study sample).

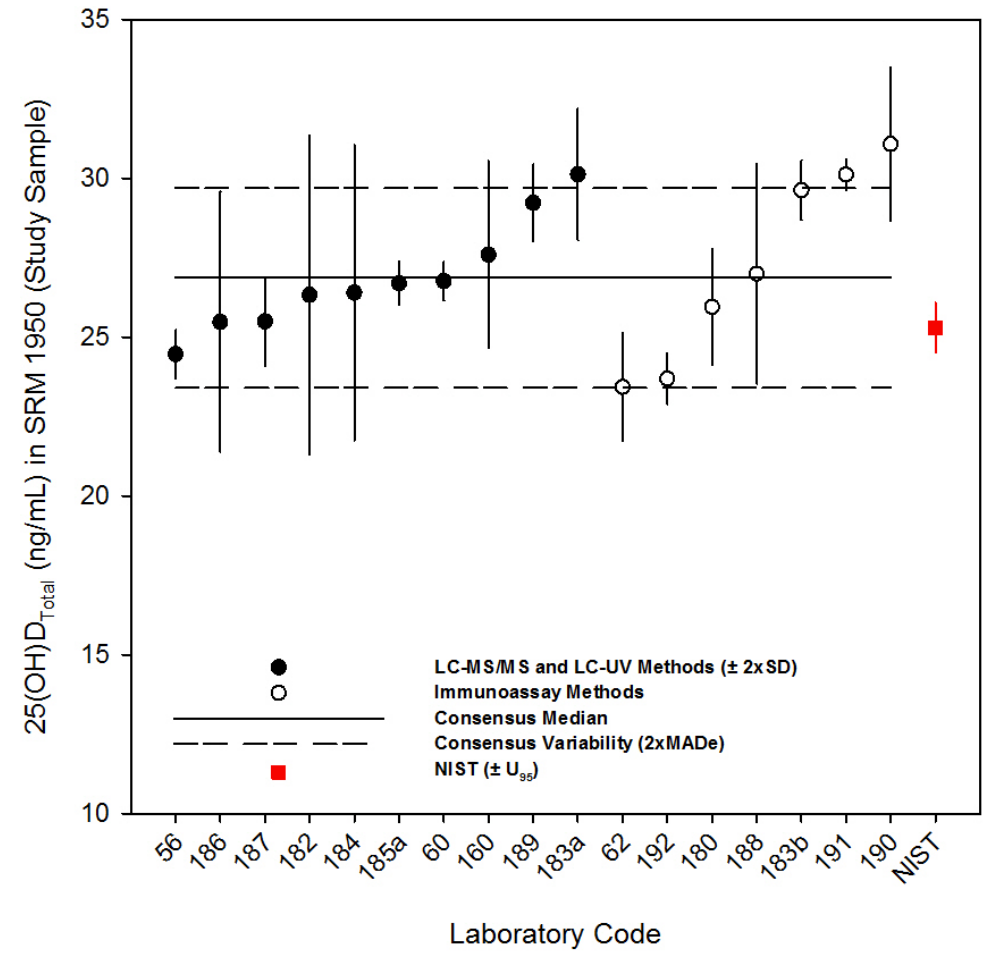


Appendix A-1. Summary of immunoassay methods used by VitDQAP Winter 2010 participants

\begin{tabular}{|c|c|c|c|}
\hline $\begin{array}{l}\text { Participant } \\
\text { Number }\end{array}$ & Immunoassay & Sample Preparation & Detection \\
\hline 62 & RIA & Samples were extracted, centrifuged & Gamma counter \\
\hline 180 & RIA & $\begin{array}{l}\text { Samples were prepared per vendor's sample extraction } \\
\text { protocol }\end{array}$ & $\begin{array}{l}1^{125} \text { detection using gamma } \\
\text { counter }\end{array}$ \\
\hline $183 b$ & CLIA & $\mathrm{n} / \mathrm{r}$ & $\mathrm{n} / \mathrm{r}$ \\
\hline 188 & EIA & $\mathrm{n} / \mathrm{r}$ & $\mathrm{n} / \mathrm{r}$ \\
\hline 190 & EIA & $\begin{array}{l}\text { Calibrators, controls and test specimens were all diluted with } \\
\text { biotin-labeled } 25(\mathrm{OH}) \text { D and analyzed in duplicate }\end{array}$ & Microplate reader \\
\hline 191 & RIA & $\mathrm{n} / \mathrm{r}$ & $\mathrm{n} / \mathrm{r}$ \\
\hline 192 & EIA & $\mathrm{n} / \mathrm{r}$ & $\begin{array}{l}\text { Software was used to convert the } \\
\text { absorbance values obtained at } \\
450 \mathrm{~nm} \text { to get the concentration }\end{array}$ \\
\hline
\end{tabular}




\section{Appendix A-2. Summary of LC methods used by VitDQAP Winter 2010 participants}

\begin{tabular}{|c|c|c|c|c|}
\hline $\begin{array}{l}\text { Participant } \\
\text { Number }\end{array}$ & $\begin{array}{l}\text { Internal Standard } \\
\text { (IS) }\end{array}$ & Sample Preparation & Chromatographic Conditions & Detection \\
\hline 56 & $\begin{array}{l}25(\mathrm{OH}) \mathrm{D}_{2}-\mathrm{d}_{3} \text { and } \\
25(\mathrm{OH}) \mathrm{D}_{3}-\mathrm{d}_{6}\end{array}$ & $\begin{array}{l}\text { Samples were extracted with } \\
\text { acetonitrile, centrifuged, then } \\
\text { filtered }\end{array}$ & $\begin{array}{l}\text { Online SPE with C8 column }(2.1 \times \\
30 \mathrm{~mm}) \text {; separation on C18 column } \\
(2.1 \times 50 \mathrm{~mm}) \text {; step gradient with } \\
\text { methanol/water; flow } 0.5 \mathrm{~mL} / \mathrm{min}\end{array}$ & $\begin{array}{l}\text { MS/MS MRM: } 25(\mathrm{OH}) \mathrm{D}_{3} \mathrm{~m} / \mathrm{z} \\
\text { 401/383; } 25(\mathrm{OH}) \mathrm{D}_{3}-\mathrm{d}_{6} \mathrm{~m} / \mathrm{z} \\
\text { 407/389; 25(OH) } \mathrm{D}_{2} \mathrm{~m} / \mathrm{z} \\
\text { 413/395; 25(OH) } \mathrm{D}_{2}-\mathrm{d}_{3} \mathrm{~m} / \mathrm{z} \\
416 / 398\end{array}$ \\
\hline 60 & $25(\mathrm{OH}) \mathrm{D}_{3}-\mathrm{d}_{6}$ & $\begin{array}{l}\text { IS solution was added to plasma } \\
(150 \mu \mathrm{L}) \text {, and proteins were } \\
\text { precipiated by addition of a ternary } \\
\text { extraction solvent. Sample } \\
\text { cetrifuged, supernatant transfered } \\
\text { to new vial, dried, and dissolved in } \\
\text { mobile phase }\end{array}$ & $\begin{array}{l}\text { C18 column ( } 3.0 \times 150 \mathrm{~mm}) ; \mathrm{A} \text { : } \\
0.05 \% \text { formic acid in water, B: } \\
0.05 \% \text { formic acid in } \\
\text { methanol/acetonitrile ( } 80 / 20, \\
\text { volume fraction); isocratic elution } \\
\text { with } 92 \% \text { B from 0-2 min, step } \\
\text { gradient to } 100 \% \text { B (2-8 min), } \\
\text { equilibration (8-13) min; flow } 0.55 \\
\mathrm{~mL} / \mathrm{min}\end{array}$ & $\begin{array}{l}\text { MS/MS (positive) MRM: } \\
25(\mathrm{OH}) \mathrm{D}_{3} \mathrm{~m} / \mathrm{z} 383 / 211 ; \\
25(\mathrm{OH}) \mathrm{D}_{3}-\mathrm{d}_{6} \mathrm{~m} / \mathrm{z} 389 / 211 ; \\
25(\mathrm{OH}) \mathrm{D}_{2} \mathrm{~m} / \mathrm{z} 395 / 270\end{array}$ \\
\hline 160 & $\begin{array}{l}25(\mathrm{OH}) \mathrm{D}_{2}-\mathrm{d}_{6} \text { and } \\
25(\mathrm{OH}) \mathrm{D}_{3}-\mathrm{d}_{6}\end{array}$ & $\begin{array}{l}\text { Samples were extracted in } \\
\text { acetonitrile, centrifuged and filtered }\end{array}$ & $\begin{array}{l}\text { 2-Dimensional separation CN } \\
\text { column (2.1x 50mm) (1st } \\
\text { Dimension), C18 column ( } 2.1 \times \\
50 \mathrm{~mm})(2 \mathrm{nd} \text { Dimension); gradient } \\
\text { with } 10 \mathrm{mmol} / \mathrm{L} \text { formic acid in water } \\
\text { and } 10 \mathrm{mmol} / \mathrm{L} \text { formic acid in } \\
\text { methanol }\end{array}$ & $\begin{array}{l}\text { MS/MS MRM: } 25(\mathrm{OH}) \mathrm{D}_{3} \mathrm{~m} / \mathrm{z} \\
401 / 365 ; 25(\mathrm{OH}) \mathrm{D}_{3}-\mathrm{d}_{6} \mathrm{~m} / \mathrm{z} \\
\text { 407/371; } 25(\mathrm{OH}) \mathrm{D}_{2} \mathrm{~m} / \mathrm{z} \\
413 / 337 ; 25(\mathrm{OH}) \mathrm{D}_{2}-\mathrm{d}_{6} \mathrm{~m} / \mathrm{z} \\
419 / 337\end{array}$ \\
\hline 182 & $25(\mathrm{OH}) \mathrm{D}_{3}-\mathrm{d}_{6}$ & $\begin{array}{l}\text { Proteins were precipitated with } \\
\text { acetonitrile/methanol (3:1) and IS } \\
\text { directly in } 96 \text { well plate. Plate was } \\
\text { covered, mixed manually, and } \\
\text { centrifuged }\end{array}$ & $\begin{array}{l}\text { C18 column }(2.1 \times 50 \mathrm{~mm}) \text {; gradient } \\
\text { elution from } 60 \%-100 \% \text { methanol } \\
\text { with formic acid and ammonium } \\
\text { acetate modifiers }\end{array}$ & $\begin{array}{l}\text { MS/MS MRM: } 25(\mathrm{OH}) \mathrm{D}_{3} \mathrm{~m} / \mathrm{z} \\
401 / 365 \text { (quant), } \mathrm{m} / \mathrm{z} \\
401 / 383 \text { (qual); } 25(\mathrm{OH}) \mathrm{D}_{2} \\
\mathrm{~m} / \mathrm{z} 413 / 355 \text { (quant), } \mathrm{m} / \mathrm{z} \\
413 / 271 \text { (qual) }\end{array}$ \\
\hline
\end{tabular}

IS $(25 \mu \mathrm{L})$ was added to plasma

$(150 \mu \mathrm{L})$, followed by protein precipiation and extraction with 0.1

$183 a \quad 25(\mathrm{OH}) \mathrm{D}_{3}-\mathrm{d}_{6} \quad \mathrm{~mol} / \mathrm{L} \mathrm{ZnSO} \mathrm{Zn}_{4}(150 \mu \mathrm{L})$, methanol extract dried and dissolved with

C8 column $(2.1 \times 50 \mathrm{~mm})$; isocratic elution with $73 \%$ methanol/ $27 \%$ water; flow $0.4 \mathrm{~mL} / \mathrm{min}$
MS/MS MRM: 25(OH) $\mathrm{D}_{3} \mathrm{~m} / \mathrm{z}$ 401/159, 401/383; 25(OH)D $D_{2}$ $\mathrm{m} / \mathrm{z}$ 413/82, 413/395 $70 \%$ methanol, 30\% water with 2 $\mathrm{mmol} / \mathrm{L}$ ammonium acetate

\begin{tabular}{|c|c|c|c|c|}
\hline 184 & $25(\mathrm{OH}) \mathrm{D}_{3}-\mathrm{d}_{6}$ & $\begin{array}{l}\text { Plasma }(200 \mu \mathrm{L}) \text { extracted with } \\
\text { acetonitrile and IS }(700 \mu \mathrm{L}) ; \text { mixed, } \\
\text { centrifuged, and filtered }\end{array}$ & $\begin{array}{l}\text { C18 column }(100 \times 2.1 \mathrm{~mm} ; 5 \mu \mathrm{m}) \\
\text { linear gradient from } 60 \% \text { B to } 98 \% \\
\text { B over } 2 \text { min (A: } 0.1 \% \text { formic acid in } \\
\text { water, B: methanol with } 0.1 \% \text { formic } \\
\text { acid and } 5 \mathrm{mmol} / \mathrm{L} \text { ammonium } \\
\text { acetate) }\end{array}$ & $\begin{array}{l}\text { MS/MS (APCI) MRM: } \\
25(\mathrm{OH}) \mathrm{D}_{3} \mathrm{~m} / \mathrm{z} 383 / 257 \\
25(\mathrm{OH}) \mathrm{D}_{3}-\mathrm{d}_{6} \mathrm{~m} / \mathrm{z} 389 / 263 \\
25(\mathrm{OH}) \mathrm{D}_{2} \mathrm{~m} / \mathrm{z} 395 / 209\end{array}$ \\
\hline 185 & $n / r$ & $n / r$ & $n / r$ & MS/MS \\
\hline 186 & $25(\mathrm{OH}) \mathrm{D}_{3}-\mathrm{d}_{6}$ & $\begin{array}{l}\text { Proteins were removed by addition } \\
\text { of } \mathrm{ZnSO}_{4} \text {, followed by methanol } \\
\text { extraction and centrifugation; } \\
\text { analytes were liquid/liquid extracted } \\
\text { with three volumes of hexane, } \\
\text { dried, and dissolved in } \\
\text { methanol/water ( } 65: 35)\end{array}$ & $\begin{array}{l}\text { Phenyl column }(2.1 \times 100 ; 1.7 \mathrm{um}) \\
40^{\circ} \mathrm{C} \text {; gradient: } 65-85 \% \text { B over } 3 \\
\text { min (A: } 0.1 \% \text { formic acid in water; B: } \\
0.1 \% \text { formic acid in methanol }) ; \text { flow } \\
0.45 \mathrm{~mL} / \mathrm{min}\end{array}$ & $\begin{array}{l}\text { MS/MS MRM: } 25(\mathrm{OH}) \mathrm{D}_{3} \mathrm{~m} / \mathrm{z} \\
401 / 159 ; 25(\mathrm{OH}) \mathrm{D}_{2} \mathrm{~m} / \mathrm{z} \\
413 / 395\end{array}$ \\
\hline 187 & $n / r$ & $n / r$ & $n / r$ & MS/MS \\
\hline 189 & $\begin{array}{l}\text { Obtained from kit } \\
\text { supplier }\end{array}$ & $\begin{array}{l}\text { Proteins were precipitated, samples } \\
\text { centrifuged, analytes in the } \\
\text { supernatant were extracted using } \\
\text { SPE cartridges }\end{array}$ & $\begin{array}{l}\text { Commercially obtained reagent set } \\
\text { and column; isocratic elution; flow } \\
0.7 \mathrm{~mL} / \mathrm{min}\end{array}$ & UV at $265 \mathrm{~nm}$ \\
\hline
\end{tabular}


Appendix B. Raw participant data for $25(\mathrm{OH}) \mathrm{D}_{2}, 25(\mathrm{OH}) \mathrm{D}_{3}$ and $25(\mathrm{OH}) \mathrm{D}_{\text {Total }}$.

\begin{tabular}{|c|c|c|c|c|c|c|c|c|c|c|c|c|}
\hline \multirow[b]{3}{*}{ Lab } & \multirow[b]{3}{*}{ Method } & \multicolumn{3}{|c|}{$25(\mathrm{OH}) \mathrm{D}_{2}(\mathrm{ng} / \mathrm{mL})$} & \multicolumn{3}{|c|}{$25(\mathrm{OH}) \mathrm{D}_{3}(\mathrm{ng} / \mathrm{mL})$} & \multicolumn{3}{|c|}{$25(\mathrm{OH}) \mathrm{D}_{\text {Total }}(\mathrm{ng} / \mathrm{mL})$} & \multirow{2}{*}{\multicolumn{2}{|c|}{$\frac{25(\mathrm{OH}) \mathrm{D}_{2} / \mathrm{D}_{3}(\mathrm{ng} / \mathrm{mL})}{\mathrm{SRM} 2972}$}} \\
\hline & & SRM 1950 & SRM 1950 & SRM 1950 & SRM 1950 & SRM 1950 & SRM 1950 & SRM 1950 & SRM 1950 & SRM 1950 & & \\
\hline & & Vial A & Vial B & Vial C & Vial A & Vial B & Vial C & Vial A & Vial B & Vial C & $25(\mathrm{OH}) \mathrm{D}_{2}$ & $25(\mathrm{OH}) \mathrm{D}_{3}$ \\
\hline 056 & LC-MS/MS & 0.5 & 0.4 & $<0.4$ & 24.4 & 23.8 & 24.3 & 24.9 & 24.2 & 24.3 & 211.3 & 260.4 \\
\hline 060 & LC-MS/MS & $<2$ & $<2$ & $<2$ & 26.5 & 26.7 & 27.1 & 26.5 & 26.7 & 27.1 & 235.0 & 324.2 \\
\hline 062 & RIA & $n / a$ & $n / a$ & $\mathrm{n} / \mathrm{a}$ & $\mathrm{n} / \mathrm{a}$ & $\mathrm{n} / \mathrm{a}$ & $\mathrm{n} / \mathrm{a}$ & 22.6 & 24.3 & 23.4 & 175.7 & 340.3 \\
\hline 160 & LC-MS/MS & $<1.0$ & $<1.0$ & $<1.0$ & 26.3 & 29.2 & 27.3 & 26.3 & 29.2 & 27.3 & 237.0 & 329.0 \\
\hline 180 & RIA & $\mathrm{n} / \mathrm{a}$ & $\mathrm{n} / \mathrm{a}$ & $\mathrm{n} / \mathrm{a}$ & $\mathrm{n} / \mathrm{a}$ & $\mathrm{n} / \mathrm{a}$ & $\mathrm{n} / \mathrm{a}$ & 25.4 & 27.0 & 25.5 & $n / r$ & $\mathrm{n} / \mathrm{r}$ \\
\hline 182 & LC-MS/MS & $<2$ & $<2$ & $<2$ & 24.0 & 26.0 & 29.0 & 24.0 & 26.0 & 29.0 & 262.0 & 368.0 \\
\hline $183 a$ & LC-MS/MS & $<4$ & $<4$ & $<4$ & 31.3 & 29.8 & 29.3 & 31.3 & 29.8 & 29.3 & 161.2 & 353.8 \\
\hline $183 b$ & CLIA & $n / a$ & $\mathrm{n} / \mathrm{a}$ & $n / a$ & $\mathrm{n} / \mathrm{a}$ & $\mathrm{n} / \mathrm{a}$ & $\mathrm{n} / \mathrm{a}$ & 30.0 & 29.1 & 29.8 & 205.0 & 291.0 \\
\hline 184 & LC-MS/MS & $<1.0$ & $<1.0$ & $<1.0$ & 23.8 & 28.3 & 27.1 & 23.8 & 28.3 & 27.1 & 223.0 & 351.0 \\
\hline $185 a$ & LC-MS/MS & $\mathrm{n} / \mathrm{d}$ & $n / d$ & $n / d$ & 26.4 & 26.7 & 27.0 & 26.4 & 26.7 & 27.0 & 201.0 & 354.4 \\
\hline 186 & LC-MS/MS & $n / r$ & $n / r$ & $n / r$ & 27.6 & 25.3 & 23.5 & 27.6 & 25.3 & 23.5 & 240.6 & 333.8 \\
\hline 187 & LC-MS/MS & 0.7 & 0.8 & 0.7 & 24.8 & 24.0 & 25.5 & 25.5 & 24.8 & 26.2 & 214.0 & 347.0 \\
\hline 188 & CLIA & $\mathrm{n} / \mathrm{a}$ & n/a & $n / a$ & $\mathrm{n} / \mathrm{a}$ & $\mathrm{n} / \mathrm{a}$ & $\mathrm{n} / \mathrm{a}$ & 28.0 & 28.0 & 25.0 & $\mathrm{n} / \mathrm{r}$ & $\mathrm{n} / \mathrm{r}$ \\
\hline 189 & LC-UV & $\mathrm{n} / \mathrm{d}$ & $n / d$ & $n / d$ & 29.8 & 29.3 & 28.6 & 29.8 & 29.3 & 28.6 & $n / r$ & $n / r$ \\
\hline 190 & EIA & $\mathrm{n} / \mathrm{a}$ & $n / a$ & $n / a$ & $\mathrm{n} / \mathrm{a}$ & $\mathrm{n} / \mathrm{a}$ & $n / a$ & 32.5 & 30.4 & 30.4 & $n / r$ & $n / r$ \\
\hline 191 & RIA & $\mathrm{n} / \mathrm{a}$ & n/a & $n / a$ & $\mathrm{n} / \mathrm{a}$ & $\mathrm{n} / \mathrm{a}$ & $n / a$ & 29.9 & 30.4 & 30.1 & 309.6 & 319.8 \\
\hline 192 & EIA & $\mathrm{n} / \mathrm{a}$ & n/a & $\mathrm{n} / \mathrm{a}$ & n/a & $\mathrm{n} / \mathrm{a}$ & $\mathrm{n} / \mathrm{a}$ & 23.5 & 23.4 & 24.2 & $n / r$ & $n / r$ \\
\hline
\end{tabular}

${ }^{*} \mathrm{n} / \mathrm{a}=$ not applicable (for immunoassay methods); $\mathrm{n} / \mathrm{r}=$ not reported; $\mathrm{n} / \mathrm{d}=$ not detected; $<\mathrm{X}=$ less than a reported quantitation limit of $\mathrm{X}$

\begin{tabular}{|l|l|l|}
\hline 0.52 & 0.52 & 0.52 \\
0.17 & 0.17 & 0.17 \\
\hline
\end{tabular}

\begin{tabular}{|c|c|c|}
\hline 24.78 & 24.78 & 24.78 \\
0.77 & 0.77 & 0.77 \\
\hline
\end{tabular}

\begin{tabular}{|c|c|c|}
\hline 25.30 & 25.30 & 25.30 \\
0.79 & 0.79 & 0.79 \\
\hline
\end{tabular}

\begin{tabular}{|c|c|}
\hline 238.6 & 334.8 \\
3.9 & 5.2 \\
\hline
\end{tabular}

